\title{
FIXED POINTS AND THEIR APPROXIMATION IN BANACH SPACES FOR CERTAIN COMMUTING MAPPINGS
}

\author{
by M. S. KHAN
}

(Received 4 March, 1980)

1. Let $X$ be a Banach space. Then a self-mapping $A$ of $X$ is said to be nonexpansive provided that $\|A x-A y\| \leq\|x-y\|$ holds for all $x, y \in X$. The class of nonexpansive mappings includes contraction mappings and is properly contained in the class of all continuous mappings. Keeping in view the fixed point theorems known for contraction mappings (e.g. Banach Contraction Principle) and also for continuous mappings (e.g. those of Brouwer, Schauder and Tychonoff), it seems desirable to obtain fixed point theorems for nonexpansive mappings defined on subsets with conditions weaker than compactness and convexity. Hypotheses of compactness was relaxed by Browder [2] and Kirk [9] whereas Dotson [3] was able to relax both convexity and compactness by using the notion of so-called star-shaped subsets of a Banach space. On the other hand, Goebel and Zlotkiewicz [5] observed that the same result of Browder [2] can be extended to mappings with nonexpansive iterates. In [6], Goebel-Kirk-Shimi obtained fixed point theorems for a new class of mappings which is much wider than those of nonexpansive mappings, and mappings studied by Kannan [8]. More recently, Shimi [12] used the fixed point theorem of Goebel-Kirk-Shimi [6] to discuss results for approximating fixed points in Banach spaces.

In this paper we wish to present some theorems on coincidence points and common fixed points for a pair of mappings defined on certain subsets of a Banach space. While some of these results can be viewed as applications of those due to Goebel-Zlotkiewicz [5] and Dotson [3], others are indeed generalizations of results due to Shimi [12]. In doing so we are motivated by the work of Jungck [7] who took a continuous mapping instead of the identity mapping to generalize the Banach fixed point theorems.

2. Let $K$ be a closed subset of a Banach space $X$. The well-known Banach Contraction Principle states that a contraction mapping of $K$ into itself has a unique fixed point. The same result holds if we assume that only some positive powers of a mapping are contraction, but it is not true for nonexpansive mappings. However, Goebel and Zlotkiewicz [5] observed that a result of Browder [2] can be extended to mappings with nonexpansive iterates by using some fixed point theorems they proved for involutions. The theorems of this section concerning the existence of coincidence points of two mappings are applications of Goebel-Zlotkiewicz's results.

THEOREM 2.1. Let $K$ be a closed and convex subset of a Banach space $X$. Let $F: K \rightarrow K, G: K \rightarrow K$ satisfy the following conditions:

(i) $F$ and $G$ commute,

(ii) $F^{2}=I, G^{2}=I$, where $I$ denotes the identity mapping,

(iii) $\|F x-F y\| \leq \alpha\|G x-G y\|$, for every $x, y \in K$ and $0 \leq \alpha<2$.

Glasgow Math. J. 23 (1982) 1-6. 
Then there exists at least one point $x_{0} \in K$ such that $F\left(x_{0}\right)=G\left(x_{0}\right)$. Further, if $0 \leq \alpha<1$, then $x_{0}$ is unique and $x_{0}=F\left(x_{0}\right)=G\left(x_{0}\right)$.

Proof. From (i) and (ii) it follows that $(F G)^{2}=I$, and by (ii) and (iii) we have $\|F G x-F G y\| \leq \alpha\|x-y\|$. Therefore by Theorem 1 of Goebel and Zlotkiewicz [5], FG has a fixed point, say $x_{0}$, in $K$. Since $F^{2}=I, x_{0}$ is a point of coincidence of $F$ and $G$. Proof of the second part is simple. This completes the proof.

Exactly as shown in Goebel and Zlotkiewicz [5], one can prove the following result.

THEOREM 2.2. If $X$ is a uniformly convex Banach space and $K$ is a closed and convex subset of $X$, then for each pair of mappings $F: K \rightarrow K$ and $G: K \rightarrow K$ satisfying conditions (i) and (ii) of Theorem 2.1 with $\alpha$ such that $\alpha \delta^{-1}(1-1 / \alpha)<\dot{4}$, there exists a point $x_{0} \in K$ which is the point of coincidence of $F$ and $G$. ( $\delta$ denotes the modulus of convexity of $X$ ).

The final result of this section reads as follows.

THeorem 2.3. Suppose $X$ is uniformly convex Banach space and $K$ is a closed, bounded and convex subset of $X$. If $F: K \rightarrow K$ and $G: K \rightarrow K$ satisfy the conditions

(1) $\left\|F^{2}(x)-F^{2}(y)\right\| \leq\|x-y\|$,

(2) $\|F(x)-F(y)\| \leq \alpha\|G(x)-G(y)\|$, where $\alpha$ is the same as in Theorem 2.2,

(3) $F$ and $G$ commute,

(4) $G^{2}=I$,

then $F$ and $G$ have at least one point of coincidence.

Proof. Let $C^{*}$ be the set of fixed points of $F^{2}$, a nonempty, closed, convex subset of $K$ (see e.g. Browder [2]). As $F, G$ commute we find that $G$ maps $C^{*}$ into itself. Also $F^{2}=I$ on $C^{*}$ and so Theorem 2.2 applies. This ends the proof.

3. In this section we prove two theorems concerning approximation of common fixed points for a pair of mappings in a general Banach space. Our results are indeed extensions of those obtained by Shimi [12] which are in turn generalizations of results due to Kannan [8] and Krasnoselskii [10]. The results obtained in Shimi [12] have been proved for a class of mappings which includes nonexpansive mappings. For references on similar results for nonexpansive mappings, see the papers quoted in Shimi [12].

Theorem 3.1. Let $X$ be a Banach space and $x_{0} \in X$ be arbitrary. Let $F$ and $G$ be mappings of $X$ into itself such that the following are true:

(a) $F$ and $G$ commute,

(b) $F(X) \subset G(X)$,

(c) $G$ is continuous and linear,

(d) for all $x, y \in X$, we have

$$
\begin{aligned}
\|F x-F y\| \leq & a\|G x-G y\|+b\{\|G x-F x\|+\|G y-F y\|\} \\
& +c\{\|G x-F y\|+\|G y-F x\|\},
\end{aligned}
$$

where $a, b, c \geq 0, a+2 b+2 c \leq 1$. 
Let $x_{0} \in X$ be arbitrary. If $\left\{x_{n}\right\}$ is a sequence in $X$ satisfying $G x_{n+1}=\frac{1}{2}\left(G x_{n}+F x_{n}\right)$, $n=0,1,2 \ldots$, and for which $\left\{G x_{n}\right\}$ converges, then there is at least one common fixed point of $F$ and $G$.

Proof. Define a mapping $F_{1}$ by setting $F_{1}(x)=\frac{1}{2}(G x+F x)$. Then $F_{1}\left(x_{n}\right)=G\left(x_{n+1}\right)$, $n=0,1,2, \ldots$. Also since $F(X) \subset G(X)$ and $G$ is linear it follows that $F_{1}$ is a selfmapping on $X, F_{1}(X) \subset G(X), F_{1} G=G F_{1}$ and the sequence $\left\{G x_{n}\right\}$ is a sequence of $G$-iteration of $x_{0}$ under $F_{1}$ (cf. Park [11]).

Now for any $x, y \in X$, we have

But

$$
\left\|F_{1} x-F_{1} y\right\|=\left\|\frac{1}{2} G x+\frac{1}{2} F x-\frac{1}{2} G y-\frac{1}{2} F y\right\| \leq \frac{1}{2}\|G x-G y\|+\frac{1}{2}\|F x-F y\| .
$$

Therefore

$$
\begin{aligned}
\|F x-F y\| & \leq a\|G x-G y\|+b\{\|G x-F x\|+\|G y-F y\|\}+c\{\|G x-F y\|+\|G y-F x\|\} \\
& \leq(a+2 c)\|G x-G y\|+(b+c)[\|G x-F x\|+\|G y-F y\|] .
\end{aligned}
$$

$$
\begin{aligned}
\left\|F_{1} x-F_{1} y\right\| & \leq \frac{1}{2}(1+a+2 c)\|G x-G y\|+(b+c)\left[\frac{\|G x-F x\|}{2}+\frac{\|G y-F y\|}{2}\right] \\
& \leq\|G x-G y\|+\frac{1}{2}\left[\left\|G x-F_{1} x\right\|+\left\|G y-F_{1} y\right\|\right] .
\end{aligned}
$$

If $\left\{G x_{n}\right\}$ converges to $u$, then for $n=1,2,3, \ldots$, we have

$$
\begin{aligned}
\left\|G\left(G x_{n+1}\right)-F_{1} u\right\| & =\left\|G F_{1} x_{n}-F_{1} u\right\|=\left\|F_{1} G x_{n}-F_{1} u\right\| \\
& \leq\left\|G\left(G x_{n}\right)-G u\right\|+\frac{1}{2}\left\|G\left(G x_{n}\right)-F_{1} G x_{n}\right\|+\frac{1}{2}\left\|G u-F_{1} u\right\| \\
& =\left\|G^{2} x_{n}-G u\right\|+\frac{1}{2}\left\|G^{2} x_{n}-G F_{1} x_{n}\right\|+\frac{1}{2}\left\|G u-G^{2} x_{n+1}\right\|+\frac{1}{2}\left\|G^{2} x_{n+1}-F_{1} u\right\| .
\end{aligned}
$$

Thus

$$
\frac{1}{2}\left\|G^{2} x_{n+1}-F_{1} u\right\| \leq\left\|G^{2} x_{n}-G u\right\|+\frac{1}{2}\left\|G^{2} x_{n}-G^{2} x_{n+1}\right\|+\frac{1}{2}\left\|G u-G^{2} x_{n+1}\right\| .
$$

Now using the continuity of $G$ and the fact that $G x_{n} \rightarrow u$, we find that

$$
\lim _{n}\left\|G^{2} x_{n+1}-F_{1} u\right\|=0 \text {. }
$$

From the inequality

$$
\left\|G u-F_{1} u\right\| \leq\left\|G u-G^{2} x_{n+1}\right\|+\left\|G^{2} x_{n+1}-F_{1} u\right\|,
$$

for $n=1,2, \ldots$, we see that $G u=F_{1} u$. Therefore $G u=F u$. Once again commutativity of $F$ and $G$ yields $G(G u)=G(F u)=F C(u)=F(F u)$. Then

$$
\begin{gathered}
\left\|F u-F^{2} u\right\| \leq a\|G u-G F u\|+b\left\{\|G u-F u\|+\left\|G F u-F^{2} u\right\|\right\} \\
+c\left\{\left\|G u-F^{2} u\right\|+\|G F u-F u\|\right\},
\end{gathered}
$$

which gives

$$
F(F u)=F(u)
$$


Also, $G(F u)=F G(u)=F(F u)=F(u)$, and so $F u$ is a common fixed point of $F$ and $G$. This completes the proof.

Remarks. (i) Putting $G=I$ in Theorem 3.1 we get a result due to Shimi [12].

(ii) Note that if $b>0$ then $F$ and $G$ will have a unique common fixed point.

Now we wish to investigate the solvability of certain non-linear functional equations in a Banach space.

THEOREM 3.2. Let $\left\{u_{n}\right\}$ be a sequence of elements in a Banach space $X$, and let $\left\{v_{n}\right\}$ be a sequence of solutions to the equation $G x-F x=G\left(u_{n}\right), n=1,2, \ldots$, where $F$ and $G$ are as in Theorem 3.1 except that $G$ need not be linear and we require $b>0$. Then if $\left\|G u_{n}\right\| \rightarrow 0$ as $n \rightarrow \infty$, the sequence $\left\{G v_{n}\right\}$ converges to the solution of the equation $F x=G x$.

Proof. Firstly we observe that

$$
\begin{aligned}
\left\|F v_{n}-F v_{m}\right\| \leq & a\left\|G v_{n}-G v_{m}\right\|+b\left\{\left\|G v_{n}-F v_{n}\right\|+\left\|G v_{m}-F v_{m}\right\|\right\} \\
& +c\left\{\left\|G v_{n}-F v_{m}\right\|+\left\|G v_{m}-F v_{n}\right\|\right\} \\
\leq & a\left\|G v_{n}-G v_{m}\right\|+b\left\{\left\|G u_{n}\right\|+\left\|G u_{m}\right\|\right\} \\
& +c\left\{\left\|G v_{n}-F v_{n}\right\|+\left\|F v_{n}-F v_{m}\right\|+\left\|G v_{m}-F v_{m}\right\|+\left\|F v_{m}-F v_{n}\right\|\right\} .
\end{aligned}
$$

So we have

$$
\left\|F v_{n}-F v_{m}\right\| \leq\left(\frac{a+b+c}{1-a-2 c}\right)\left[\left\|G u_{n}\right\|+\left\|G u_{m}\right\|\right]
$$

Now clearly,

$$
\begin{aligned}
\left\|G v_{n}-G v_{m}\right\| & \leq\left\|G v_{n}-F v_{n}\right\|+\left\|F v_{n}-F v_{m}\right\|+\left\|F v_{m}-G v_{m}\right\| \\
& \leq\left\|G u_{n}\right\|+\left(\frac{a+b+c}{1-a-2 c}\right)\left[\left\|G u_{n}\right\|+\left\|G u_{m}\right\|\right]+\left\|G u_{m}\right\| .
\end{aligned}
$$

Hence $\left\{G v_{n}\right\}$ is a Cauchy sequence and so it will converge to some point, say $u$. We further note from the inequality

$$
\left\|u-F v_{n}\right\| \leq\left\|u-G v_{n}\right\|+\left\|G v_{n}-F v_{n}\right\|=\left\|u-G v_{n}\right\|+\left\|G u_{n}\right\|,
$$

that $\left\{F v_{n}\right\}$ also converges to $u$. Now

$$
\begin{aligned}
\|G u-F u\| \leq & \left\|G u-G F v_{n}\right\|+\left\|F G v_{n}-F u\right\| \\
\leq & \left\|G u-G\left(F v_{n}\right)\right\|+a\left\|G^{2} v_{n}-G u\right\| \\
& +b\left\{\left\|G^{2} v_{n}-F G v_{n}\right\|+\|G u-F u\|\right\} \\
& +c\left\{\left\|G^{2} v_{n}-F u\right\|+\left\|G u-F G v_{n}\right\|\right\} .
\end{aligned}
$$

Hence

$$
(1-b-c)\|F u-G u\| \leq(1+b+c)\left\|G u-G F u_{n}\right\|+(a+b+c)\left\|G^{2} v_{n}-G u\right\| .
$$

Letting $n \rightarrow \infty$ we have $G u=F u$ as required. 
Remarks. (i) For $G=I$ our Theorem 3.2 reduces to that of Shimi [12].

(ii) As in the proof of Theorem 3.1 it follows that $F u$ is a common fixed point of $F$ and $G$. Also $F u$ will be unique since $b>0$.

4. Now we present results on fixed point theorems for a pair of mappings defined on certain special subsets of a Banach space. Our results are related to those contained in Dotson [3] who relaxed the conditions of convexity and compactness from the similar results for nonexpansive mappings obtained by Browder [2] and Kirk [9].

We introduce the following definition.

DefinITION 4.1. Let $G$ be a continuous mapping on a Banach space $X$. Then a self-mapping $F$ of $X$ is said to be $G$-nonexpansive if

$$
\|F x-F y\| \leq\|G x-G y\|,
$$

for all $x, y \in X$.

We observe that a $G$-nonexpansive mapping is always continuous.

The following definition is taken from Dotson [3].

Definition 4.2. If $C$ is a subset of a Banach space $X$, then a mapping $S: C \rightarrow X$ is called demiclosed if $\left\{x_{n}\right\} \subset C,\left\{x_{n}\right\}$ converges weakly to $x \in C$ and $S x_{n} \rightarrow y \in X$ imply that $y=S x$.

Obviously all weakly continuous maps are demiclosed.

The following is an easy consequence of results obtained by Dotson [3]. satisfy

THEOREM 4.3. Let $F, G$ be self mappings of a subset $C$ of a Banach space $X$ which

(i) $G^{2}=G$,

(ii) $F G(C) \subset G(C)$,

(iii) $F$ is $G$-nonexpansive on $C$.

Suppose $G(C)$ is star-shaped and weakly compact and that $I-F$ is demiclosed. Then $F$ and $G$ have a common fixed point. If $G(C)$ is compact, the restriction that $I-F$ be demiclosed can be dropped.

Proof. $F$ is non-expansive on $G(C)$ and so, by Theorem 2 (or Theorem 1) of Dotson [3], $F$ has a fixed point $u=G(v)$ in $G(C)$. Then $G u=G^{2} v=G v=u$ so that $u$ is a common fixed point.

Acknowledgement. I am grateful to Professor T. N. Shimi for providing me with a copy of his paper which helped to motivate the present study. Thanks are also due to the learned referee whose most valuable comments improved the contents of the paper.

\section{REFERENCES}

1. B. Brosowski, Fixpunktsätze in der Approximationstheorie, Mathematica (Cluj), 11 (1969), 195-220. 
2. F. E. Browder, Nonexpansive nonlinear operators in Banach space, Proc. Nat. Acad. Sci. U.S.A. 54 (1965), 1041-1044.

3. W. G. Dotson, Jr., Fixed point theorems for nonexpansive mappings on starshpaed subsets of Banach spaces, J. London Math. Soc., (2) 4 (1972), 408-410.

4. M. Edelstein, On fixed and periodic points under contractive mappings, J. London Math. Soc., 37 (1962), 74-79.

5. K. Goebel and E. Zlotkiewicz, Some fixed point theorems in Banach spaces, Colloq. Math., 23 (1971), 103-106.

6. K. Goebel, W. A. Kirk and T. N. Shimi, A fixed point theorem in uniformly convex spaces, Boll. Un. Mat. Ital., (4) 7 (1973), 67-75.

7. G. Jungck, An iff fixed point criterion, Math. Mag., 49 (1976), 32-34.

8. R. Kannan, Some results on fixed points III, Fund. Math., 70 (1971), 169-177.

9. W. A. Kirk, A fixed point theorem for mappings which do not increase distances, Amer. Math. Monthly, 72 (1965), 1004-1006.

10. M. A. Krasnoselskii, Two remarks about the method of successive approximations, Uspehi Mat. Nauk., 63 (1955), 123-127.

11. S. Park, Fixed points of f-contractive maps, Rocky Mountain J. Math., 8 (1978), 743-750.

12. T. N. Shimi, Approximation of fixed points of certain nonlinear mappings, J. Math. Anal. Appl., 65 (1978), 565-571.

13. S. P. Singh, An application of a fixed-point theorem to approximation theory, J. Approximation Theory, 25 (1979), 89-90.

Department of Pure Mathematics

Present address:

LA Trobe UNIVERSITY

Department of Mathematics,

BUNDOORA, VICTORIA

Australia 3083

Aligarh MusLim UNIVERSTTY,

AligARH 202001

INDIA 